AIAA 2000-3959

\title{
A THREE-DIMENSIONAL PREDICTIVE ENTRY GUIDANCE APPROACH
}

\author{
K. D. Mease* and D. T. Chen ${ }^{\dagger}$ \\ Department of Mechanical and Aerospace Engineering \\ University of California, Irvine, CA 92697 \\ S. Tandon; D. H. Young§, and S. Kim $₫$ \\ The Boeing Company \\ 5301 Bolsa Avenue \\ Huntington Beach, CA 92647
}

\begin{abstract}
The Space Shuttle's two-dimensional entry trajectory planning method is extended to three dimensions. Both angle-of-attack and angle-of-bank variations are used to control the entry trajectory. The trajectory planning is done with a third-order system of differential equations using the drag and lateral accelerations as intermediate controls. The reduced-order planning problem is solved first in a simple manner with a fixed angle-ofattack profile and second as an optimal control problem. The capability is demonstrated for planning a trajectory so that a desired final heading angle is achieved. A method for extracting dynamically consistent values for the state and control variables not involved in the reduced-order planning is presented. Finally, a comparison with optimal solutions to a full-order entry problem is given, illustrating that the solution from the reduced-order planning has desirable features as a reference trajectory for tracking.
\end{abstract}

\section{Introduction}

Next generation reusable launch vehicles (RLVs) and orbital transfer vehicles (OTVs) will benefit from a greater level of autonomy and capability relative to current reusable launch vehicles. In this paper, further developments of an onboard entry trajectory planning approach ${ }^{1}$ are presented. The entry trajectory planning onboard the U.S. Space Shuttle orbiter considers only the longitudinal motion in planning a drag profile that will achieve the desired downrange. ${ }^{2}$ Our approach considers longitudinal and lateral motion and generates a three-dimensional (3D) trajectory that achieves the desired downrange and crossrange. The planning method, based on a reduced-order model of the translational motion, generates drag and lateral acceleration profiles. For guidance, the profiles would be flown using a tracking law to generate angleof-attack and angle-of-bank commands to the control system. An appropriate tracking law has been presented elsewhere. ${ }^{4}$ The profiles can also be revised during flight using the same planning method.

* Professor; Associate Fellow AIAA

$\dagger$ Graduate Research Assistant

¥Senior Engineer/Scientist; Member AIAA

$\S$ Senior Manager; Member AIAA

๑Engineer/Scientist; Senior Member AIAA

Copyright (C) 2000 by the authors. Published by the American Institute of Aeronautics and Astronautics, Inc. with permission.

\section{Entry Planning Problem}

\section{Entry Dynamics}

Energy is used in place of time as the independent variable. The energy $E$ is defined by

$$
E=\frac{1}{2} V^{2}-\left(\frac{\mu}{r}-\frac{\mu}{r_{s}}\right)
$$

where $V$ is the velocity magnitude, $r$ and $r_{s}$ are the radial distances from the planet center to the vehicle center of mass and the planet surface, respectively, and $\mu$ is the gravitational constant.

Denoting $d(\cdot) / d E$ by $(\cdot)^{\prime}$ and using $(\cdot)^{\prime}=$ $(1 / \dot{E}) d(\cdot) / d t$ and $\dot{E}=-D V$, the translational equations of motion ${ }^{5-7}$ for the center of mass of an unpowered vehicle of constant mass flying over a nonrotating spherical planet with a stationary atmosphere take the form

$$
\begin{aligned}
\theta^{\prime} & =-\frac{\cos \gamma \cos \psi}{r \cos \phi}\left(\frac{1}{D}\right) \\
\phi^{\prime} & =-\frac{\cos \gamma \sin \psi}{r}\left(\frac{1}{D}\right) \\
\psi^{\prime} & =\left[\frac{\cos \psi \tan \phi}{r}\left(\frac{1}{D}\right)-\frac{1}{V^{2}}\left(\frac{L \sin \sigma}{D}\right)\right] \cos \gamma \\
r^{\prime} & =-\sin \gamma\left(\frac{1}{D}\right) \\
\gamma^{\prime} & =\left(g-\frac{V^{2}}{r}\right) \frac{\cos \gamma}{V^{2}}\left(\frac{1}{D}\right)-\frac{1}{V^{2}}\left(\frac{L}{D} \cos \sigma\right)
\end{aligned}
$$


where $\theta$ is the longitude, $\phi$ is the latitude, $\gamma$ is the flight path angle, and $\psi$ is the heading angle with $\psi=0$ corresponding to flight due east. The bank angle $\sigma$ is defined such that the lift vector is in the vertical plane at zero bank. The acceleration due to gravity is $g=\mu / r^{2}$. $L$ and $D$ represent the lift and drag accelerations (specific forces) and are given by

$$
\begin{aligned}
L & =\frac{1}{2} \rho(r) V^{2} \cdot \frac{S}{m} \cdot C_{L}(\alpha, V, r) \\
D & =\frac{1}{2} \rho(r) V^{2} \cdot \frac{S}{m} \cdot C_{D}(\alpha, V, r)
\end{aligned}
$$

where $\rho(r)$ is the density as a function of the altitude, $C_{L}(\alpha, V, r)$ and $C_{D}(\alpha, V, r)$ are the lift and drag coefficients, $S$ is the reference area, and $m$ is the vehicle mass. There are only five equations, because with energy as the independent variable the velocity $V$ can be determined from $r$ and $E$.

\section{Vehicle and Control Constraints}

The vehicle constraints on the maximum dynamic pressure, aerodynamic acceleration, and heating rate are given by

$$
\begin{gathered}
q=\frac{1}{2} \rho V^{2} \leq q_{\max } \\
A=\left(D^{2}+L^{2}\right)^{1 / 2}=D(1+L / D)^{1 / 2} \leq A_{\max } \\
\dot{Q}=c \sqrt{\rho} V^{3} \leq \dot{Q}_{\max }
\end{gathered}
$$

The angle-of-attack $\alpha$ and the angle-of-bank $\sigma$ are taken to be the controls. There may be restrictions on the values of both controls. In this paper we only consider energy-dependent bounds on $\alpha$; namely, we require that $\alpha(E) \in\left[\alpha_{\min }(E), \alpha_{\max }(E)\right]$.

\section{Problem Statement}

To simplify notation, let $x=(\theta, \phi, \psi, r, \gamma)$ denote the state. The entry dynamics are expressed concisely as $x^{\prime}=f(x, \alpha, \sigma)$. The target longitude $\theta_{f}$ and latitude $\phi_{f}$ are given at a specified final energy $E_{f}$; these specifications are represented as terminal equality constraints $\Gamma\left(x\left(E_{f}\right)\right)=\left(\theta\left(E_{f}\right)-\theta_{f}, \phi\left(E_{f}\right)-\phi_{f}\right)^{T}=$ $(0,0)^{T}$. We note that the final heading angle could be specified also. We assume there is an allowable interval of final altitudes and our trajectory planning approach addresses this requirement, but we do not include this requirement in our problem statement.

The entry trajectory planning problem is: given the state $x\left(E_{0}\right)$ at an initial energy $E_{0}$, the terminal constraints, and the vehicle constraints, determine feasible controls $(\alpha(E), \sigma(E))$ on the interval $\left[E_{0}, E_{f}\right]$. Feasible means that the state trajectory and the controls satisfy the boundary conditions and the vehicle and control constraints.

\section{Soft Constraints}

In cases where there are multiple solutions to the entry problem, one can impose additional specifications to distinguish and choose between them. At each energy and each magnitude of the bank angle, there is a minimum lift

$$
(L \cos \sigma)_{\text {min }}=\left(g-\frac{V^{2}}{r}\right)
$$

dictated by the equilibrium glide boundary; below this minimum lift the vehicle cannot generate enough lift force to balance the effective weight. For each value of $\alpha$, there is a maximum altitude that corresponds to the equilibrium glide boundary. The vehicle does not have enough control authority to sustain level flight above this altitude, although transient excursions are possible.

In addition to keeping the heating rate $\dot{Q}$ below a specified upper limit it may be desirable to minimize the heat load

$$
Q=\int_{E_{0}}^{E_{f}} \dot{Q}\left(\frac{d E}{d t}\right)^{-1} d E
$$

depending on the type of thermal protection used for the vehicle. Minimizing the heat load is accomplished by flying on the active vehicle constraint boundary dynamic pressure, heating rate, or aerodynamic acceleration - for much of the trajectory, which shortens the flight time. On the other hand, to accommodate dispersions it is desirable for the trajectory to be away from the constraint boundaries. The most desirable entry trajectory is some compromise between minimizing the heat load and leaving margin for dispersions.

\section{Coordinate Redefinition}

Because we are not accounting for planet rotation, the "equatorial" plane can be defined as any plane containing the planet center. For trajectory planning when the initial and target positions are given, it is convenient to define the equatorial plane as the plane containing the initial position of the vehicle (more precisely the center of mass position) and the target position, with the "north pole" oriented so that the shortest distance to the target is in the easterly direction. Henceforth, we shall refer to this plane as the target plane. The initial and target positions will always have $\phi=0$, which is the equation for the target plane. The corresponding heading angle is measured from the direction corresponding to "east" in this target coordinate frame.

\section{Space Shuttle 2D Trajectory Planning}

The entry guidance for the Space Shuttle ${ }^{2}$ is divided into three phases: hypersonic, terminal area energy management (TAEM), and approach and landing. The entry guidance targets to a desired TAEM interface condition during the hypersonic phase; this condition is specified by a longitude, latitude, altitude, and a speed. The specified speed is about Mach 2. 
(What we are calling the hypersonic entry phase actually includes supersonic flight.) Note that, in general, the length $s$ of a trajectory is given by

$$
\begin{aligned}
s & =\int_{0}^{t_{f}} V d t=-\int_{E_{0}}^{E_{f}} \frac{d E}{D(E)} \\
& =-\int_{V_{0}}^{V_{f}} \frac{d V}{D+g \sin \gamma}
\end{aligned}
$$

If the required trajectory length were known, a drag acceleration profile could be specified that is consistent with this trajectory length. The onboard trajectory planning for the Space Shuttle assumes that the hypersonic portion of the entry trajectory is a great circle arc extending from the radial line containing the initial or current vehicle position to the radial line containing the desired TAEM interface position. The radial distance (from the Earth center) corresponding to the TAEM interface altitude, denoted by $r_{f}$, is the assumed radius of the great circle arc. By representing $D(E)$ as a piecewise quadratic function of $E$, the Space Shuttle trajectory planning is done analytically. (For most of the drag segments, $V$ rather than $E$ is used as the independent variable in the Space Shuttle formulation. Because $\gamma=0$ for the assumed great circle arc, the relation between trajectory length and drag is equally simple for the two choices of independent variable. For flight paths for which $g \sin \gamma$ is not negligible compared to $D$, the independent variable $E$ is preferred.)

Flying an arbitrary drag acceleration profile may cause violations of the constraints. Fortunately, the vehicle constraints can all be represented as drag acceleration constraints. An entry corridor can be plotted in the drag versus energy plane. At each energy, there is a maximum drag dictated by the active vehicle constraint. There is also a minimum drag dictated by the zero bank equilibrium glide condition

$$
L=\left(g-\frac{V^{2}}{r}\right)
$$

The Space Shuttle trajectory planning assumes a fixed angle-of-attack profile. With $\alpha$ given as a function of $E$ and $r=r_{f}$, there is a unique value of $L / D$ for each value of $E$. Thus we have

$$
D_{\min }=L_{\min }\left(\frac{L}{D}\right)^{-1}=\left(g-\frac{V^{2}}{r}\right)\left(\frac{L}{D}\right)^{-1}
$$

If $D$ is less than $D_{\min }$, there is insufficient lift to achieve $\gamma^{\prime} \geq 0$, indicating a lack of maneuverability. By selecting a drag profile that lies within the entry corridor, satisfaction of the vehicle constraints and maneuverability to accommodate dispersions is assured.

Once the drag profile has been determined, the other trajectory and control information, consistent with this drag profile, can be computed. The first and second derivatives of the drag acceleration with respect to energy provide algebraic relations satisfied by the state and control variables. At each energy, with the angle-of-attack given, $r$ can be extracted from the drag model. The $D^{\prime}$ and $D^{\prime \prime}$ equations can then be solved for $\gamma$ and $(L / D) \cos \sigma$. For tracking, $(L / D) \cos \sigma$ is viewed as the commanded variable; bank angle modulation is the primary means of achieving the command, but $\alpha$ modulation is also used as a secondary means.

In terms of Eqs. (2), the Space Shuttle planning can be viewed as follows. The great circle arc assumption used for the Space Shuttle corresponds to the conditions $\psi=0, \phi=0, \cos \gamma=1$ and $r=r_{f}$, and the first equation of Eqs. (2) reduces to

$$
\theta^{\prime}=-\frac{1}{r_{f}}\left(\frac{1}{D}\right)
$$

and $\theta$ is the angular displacement along the great circle arc, or one can use distance variable $R=r_{f} \theta$.

In general, flying the planned drag acceleration profile will require $|(L / D) \cos \sigma|<L / D$, and hence, $(L / D) \sin \sigma \neq 0$ and the heading angle will change. Furthermore, the initial (or current) heading angle may not be zero as assumed. Consequently, there will typically be lateral motion and $\phi$ will not be zero. The Space Shuttle guidance handles this lateral motion by defining a heading angle corridor and commanding a bank reversal when a boundary of this corridor is reached. The lateral motion is thus minimized to the degree that it can be neglected in planning the drag. In the following, we develop a planning procedure that allows for entry trajectories with significant lateral motion.

\section{Reduced-Order 3D Trajectory Planning}

The direct generalization of the Space Shuttle planning starts with the assumption that the hypersonic entry flight path evolves on the surface of a sphere, with center coinciding with the Earth's center. Let the radius of the sphere be given by $\hat{r}$. At a minimum, we would need to employ the differential equations for longitude and latitude to plan the motion on this sphere. In addition to drag, we could treat the heading angle $\psi$ as a variable to be specified. However, it is not clear what values of $\psi$ would lead to a feasible entry trajectory. By including the differential equation for the heading angle, we can instead treat the lateral acceleration $L \sin \sigma$ as the second variable to be specified. While conceptually we view the drag and lateral acceleration components of the total aerodynamic acceleration (or specific force) as the variables we plan, the variable $(L / D) \sin \sigma$ is a little more convenient than $L \sin \sigma$ and we shall use it. 


\section{Reduced-Order System}

The reduced-order system for trajectory planning is

$$
\begin{aligned}
\theta^{\prime} & =-\frac{\cos \psi}{\hat{r} \cos \phi}\left(\frac{1}{D}\right) \\
\phi^{\prime} & =-\frac{\sin \psi}{\hat{r}}\left(\frac{1}{D}\right) \\
\psi^{\prime} & =-\frac{1}{V^{2}}\left(\frac{L \sin \sigma}{D}\right)+\frac{\cos \psi \tan \phi}{\hat{r}}\left(\frac{1}{D}\right)
\end{aligned}
$$

Although we used flight on the surface of a fixed radius sphere to motivate our approach, we introduce an additional degree of flexibility by allowing $\hat{r}$ to be a known function of $E$, not necessarily constant. We do assume that the flight path angle is sufficiently small that setting $\cos \gamma=1$ causes a negligible error. It is important to emphasize that the approximations, $r=\hat{r}$ and $\cos \gamma=1$, are only used when evaluating $\theta^{\prime}, \phi^{\prime}$ and $\psi^{\prime}$. We have implicitly defined the small parameter $\epsilon=\Delta r / \hat{r}$, where $\Delta r=r-\hat{r}$, employed the expansions $r^{-1}=\hat{r}^{-1}(1+\epsilon+\ldots)$ and $\cos \gamma=1-\gamma^{3} / 6+\ldots$, and neglected the $O\left(\epsilon, \gamma^{3}\right)$ terms in the differential equations for $\theta, \phi$ and $\psi$. Once $\theta, \phi$, $\psi, D$, and $(L / D) \sin \sigma$ are determined, an extraction algorithm, described later, will be used to determine the corresponding values of $r, \gamma, \alpha$ and $\sigma$. The extracted values of $r$ and $\gamma$ in general will not be $\hat{r}$ and 0 , respectively.

\section{Admissible Control Set}

We need to define the admissible set of values of the intermediate controls $D$ and $(L / D) \sin \sigma$. The first step is to express the vehicle constraints as constraints on the drag acceleration. The dynamic pressure, aerodynamic acceleration, and heating rate constraints take the form

$$
\begin{gathered}
D \leq q_{\max } S C_{D}(\alpha, E, \hat{r}) \\
D \leq A_{\max }\left(1+\frac{L}{D}(\alpha, E, \hat{r})\right)^{-1 / 2} \\
D \leq \dot{Q}_{\max } \frac{C_{D} S}{2 m c^{2} V^{2(k-1)}}
\end{gathered}
$$

We approximate $r$ by $\hat{r}$ in these constraints. Then at each value of the energy in the range $\left[E_{0}, E_{f}\right]$, the drag constraints depend only on $\alpha$. The admissible set of values for the controls $(D,(L / D) \sin \sigma)$ is generated at each value of $E .^{1}$ An example of the admissible set for a particular value of $E$ is shown in Fig. 1.

\section{Feasible Trajectory Planning}

A simple method for entry trajectory planning, using the reduced-order system, follows from the assumptions of a fixed $\alpha$ profile and equilibrium glide flight. The method is initialized by estimating the required trajectory length with a great circle arc entry trajectory at $\bar{r}=\left(r_{f}+r_{0}\right) / 2$. A drag profile then

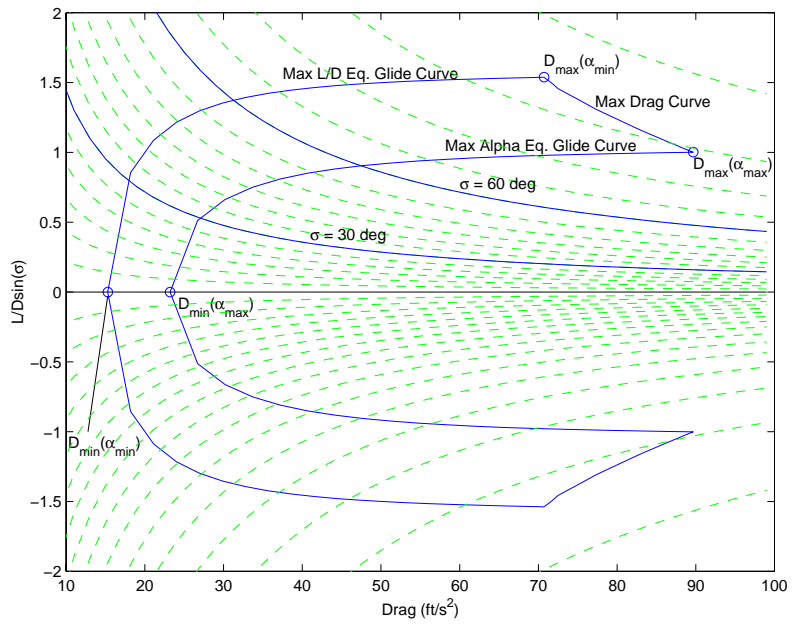

Fig. 1 Admissible control set.

is estimated from the trajectory length. The drag profile is represented by three linear spline segments with respect to energy. The endpoints of the profile are fixed to desired initial and final drag values and the second segment is specified as a constant drag profile. With these constraints and continuity requirements, the estimated drag profile is determined by a one parameter search. The parameter is determined such that the required trajectory length is achieved. The vehicle constraints for the fixed $\alpha$ profile can be satisfied by constraint arcs or adjusting additional parameters in the drag profile representation. Assuming a single bank reversal, modeled either by an instantaneous change in bank angle sign or a finite duration canned maneuver, the bank reversal initiation time can be adjusted iteratively until the integration of the reduced-order system produces a sufficiently accurate final longitude and latitude. Equilibrium glide flight requires that

$$
\frac{L}{D} \cos \sigma=\frac{1}{D}\left[g(\hat{r})-\frac{V^{2}}{\hat{r}}\right]
$$

and hence the lateral component of $L / D$ can be determined with

$$
\frac{L}{D} \sin \sigma=\left[\left(\frac{L}{D}\right)^{2}-\left(\frac{L}{D} \cos \sigma\right)^{2}\right]^{1 / 2}
$$

Using the $\alpha$ profile and $r=\hat{r}, L / D$ can be determined and then the normal and lateral components of lift can be determined. The estimated drag profile can be extended, using extrapolation, when additional arc length is needed during the iterations. The additional arc length is extrapolated with the equation

$$
s_{i+1}=s_{i}+\left(s_{0}-\bar{r} \theta\left(E_{f}\right)\right)
$$

where $s_{0}$ is the initial estimate of the arc length, based on the great circle arc assumption. The stopping criterion for the iterations is that the final errors in $\theta$ and $\phi$ 
are within tolerances or that the solution stops improving. Once the iteration has converged, the required arc (i.e., trajectory) length is known and a drag profile consistent with this arc length can be constructed within the constraints.

The most difficult requirements of the simple planning method are the one parameter searches for the drag profile and bank reversal. This simple planning method will generate a feasible trajectory that can either be used directly or as a starting solution for the optimal trajectory planning described in the next subsection.

\section{Optimal Trajectory Planning}

The entry trajectory planning problem, based on the reduced-order system, can be posed and solved as an optimal control problem of the following form: Minimize the performance index

$$
\begin{aligned}
J= & \Phi\left(x\left(E_{f}\right), E_{f}\right)+ \\
& \int_{E_{0}}^{E_{f}} \mathcal{L}(x(E), u(E), v(E), E) d E
\end{aligned}
$$

subject to the following conditions:

- the differential constraints

- initial state conditions

$$
x(0)=x_{0}
$$

- the terminal state conditions

$$
\Psi\left(x\left(E_{f}\right), E_{f}\right)=0
$$

- the algebraic state and control constraints

$$
g(x(E), u(E), v(E)) \geq 0, \forall E \in\left[E_{0}, E_{f}\right]
$$

where $x=(\theta, \phi, \chi), u=\left(u_{1}, u_{2}\right)=(D, L / D \sin \sigma)$, and $v=\left(v_{1}, v_{2}\right)$. Along with the three equations of motion, the two additional differential equations $u_{1}^{\prime}=v_{1}$ and $u_{2}^{\prime}=v_{2}$ are included in the differential constraints.

A variety of specific formulations are possible, depending on whether the various constraints are handled as soft (i.e., in the performance index) or hard (as equality or inequality constraints).

A versatile performance index is defined by

$$
\begin{aligned}
\Phi= & a_{1}\left(\chi\left(E_{f}\right)-\chi_{f}\right)^{2} \\
\mathcal{L}= & a_{2} \frac{V}{\sqrt{D}}+a_{3}\left(v_{1}(E)^{2}+v_{2}(E)^{2}\right) \\
& +a_{4}\left(b_{1} F_{1}^{2}+b_{2} \frac{1}{F_{2}}+b_{3} F_{3}\right)
\end{aligned}
$$

where $a_{1}$ through $a_{4}$ and $b_{1}$ through $b_{2}$ are nonnegative constants. The constraints on the final longitude and latitude are enforced as equality constraints which eliminates the need for soft constraints on longitude and latitude. The final heading angle and heat load are treated as soft constraints. Thus, only the heading angle appears in $\Phi$ and, noting that $V / \sqrt{D}$ is proportional to $d Q / d E,{ }^{8}$ the heat load is included in $J$. The constraints on $v_{1}$ and $v_{2}$ are enforced as both hard and soft constraints. The functions $F_{1}$, $F_{2}$, and $F_{3}$ are representative of the maximum drag, $\alpha_{\max }$ equilibrium glide, and $(L / D)_{\max }$ equilibrium glide constraint curves of the admissible set and constitute the boundaries of the admissible set as shown in Fig. $2 .^{1}$ The constraints on the admissible set are thus given by its three boundaries. The two equilibrium glide boundaries are treated as soft constraints, while the maximum drag boundary is treated as both a hard and soft constraint. Figure 2 shows how the three dimensional corridor is approximated by the polynomial fits of $P_{n}^{(1)}-P_{n}^{(6)}$ and $P_{1}$ as functions of energy. These polynomials allow the functions $F_{1}, F_{2}$, and $F_{3}$ to vary accordingly with energy.

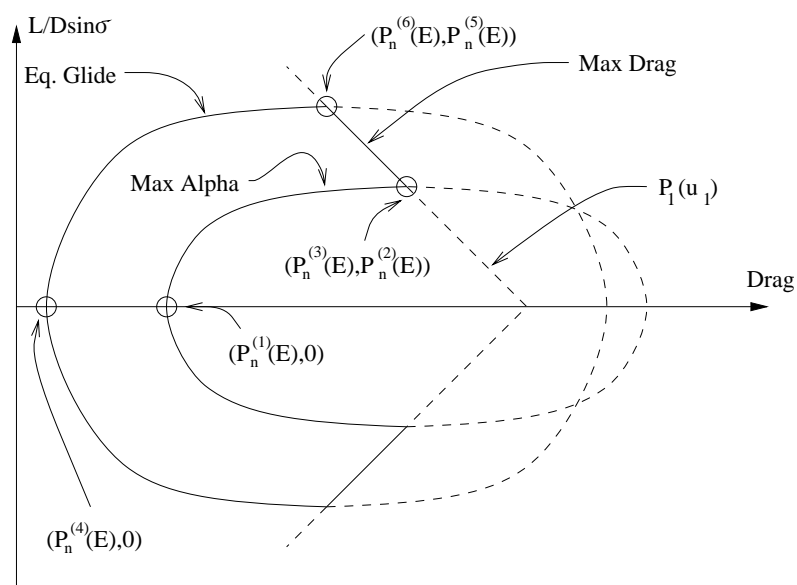

Fig. 2 Illustration of approximated sections for the admissible set boundaries.

\section{Determining Remaining States and Controls}

We used a reduced-order model to simplify, and reduce the numerical sensitivity of, the trajectory computation. The reduced-order trajectory planning determines $\theta, \phi, \chi, D$, and $(L / D) \sin \sigma$ as functions of $E$. The remaining state and control variables can be determined from this information and the differential equations.

Differentiating the model for the drag acceleration, with respect to $E$, and neglecting derivatives of the drag coefficient, yields

$$
D^{\prime}=\frac{2 D}{V^{2}}+\left(\frac{2 g}{V^{2}}+\frac{1}{H}\right) \sin \gamma
$$

Since the control rate $D^{\prime}$ is calculated in the optimal control problem, there is enough information with $r(E)=\hat{r}(E)$ to determine $\gamma(E)$. Using a backward finite difference on $\gamma$ to approximate $\gamma^{\prime}\left(E_{0}\right)$, we then have four algebraic equations from which to extract the four unknowns $r, \gamma, \alpha$, and $\sigma$ for the initial energy $E_{0}$. Future values of $r$ and $\gamma$ are determined by 
integrating the $r^{\prime}$ and $\gamma^{\prime}$ equations. The controls $\alpha$ and $\sigma$ are then calculated algebraically at each value of $E$. The dynamic consistency of the extracted controls and states can be checked and updated with a full-state simulation.

To perform the full-state simulation, the controls along with an initial altitude and flight path angle from the extraction algorithm are used to integrate the five equations of motion (2). To account for errors in the pseudo-controls, a feedback control is added to track the reference $D$ and $(L / D) \sin \sigma$ profiles. The controller will adjust the angle of attack and bank angle based on the error in drag. The angle-of-attack and bank angle are modulated by the equations

$$
\begin{aligned}
\Delta \alpha & =\frac{C_{D}\left(D_{r}-D\right)}{k_{1}} \\
\Delta \sigma & = \pm\left(k_{2} \Delta \alpha-k_{3}\left(D_{r}^{\prime}-D^{\prime}\right)\right)
\end{aligned}
$$

where the sign of $\Delta \sigma$ is determined by the sign of $\sigma$. The noise introduced by numerical differentiation limits the gain on $k_{3}$ such that an insignificant amount of damping is available. Filtering of the $D^{\prime}$ information may allow significantly higher gains to be used.

\section{OTIS}

The Boeing Company has developed Optimal Trajectories by Implicit Simulation (OTIS) ${ }^{9}$ to provide a general purpose FORTRAN program for simulating and optimizing trajectories of a wide variety of aerospace vehicles. The program is designed to simulate and optimize trajectories of launch vehicle, aircraft, missiles, satellites and interplanetary vehicles with provisions made for free and fixed end constraints, specified way-points, and path constraints.

OTIS is primarily a three degree of freedom (point mass, 3 DOF) simulation program for single vehicles. Options allow for six degree of freedom (6 DOF) simulations and several types of multiple vehicles problems.

\section{Results and Discussion}

As a first step in assessing the efficacy of our planning approach, we discuss and present results that were obtained by solving the optimal control problem with a general purpose optimization code: the Sparse Optimal Control Software (SOCS). ${ }^{10}$ We envision that a special purpose optimization algorithm would be used for the onboard implementation of our approach for faster computation and reduced code size.

SOCS solves the optimal control problem using a direct transcription method. State and control variables are discretized to reduce the optimal control problem into a nonlinear programming problem. The solution to the sparse nonlinear program is then determined using sequential quadratic programming. SOCS is capable of generating a linear initial guess from given initial and final controls, or a subroutine can be written to generate an initial guess.

\section{Reduced-Order 3D Planning Algorithm}

The reduced-order planning algorithms for the various formulations were coded primarily in FORTRAN. The algorithms were tested and evaluated on a Pentium II $300 \mathrm{MHz} \mathrm{PC}$ with $64 \mathrm{Mb}$ of RAM running Windows 95. The most significant source of difficulty and computation time, for SOCS, was the hard constraints for the admissible set. This formulation typically required 2 to 5 minutes of computation time, which did not guarantee a feasible solution from SOCS. The addition of soft constraints on the admissible set to the performance index, improved the reliability of the program by keeping solutions away from the nonconvex $\alpha_{\max }$ equilibrium glide boundary. Once the hard constraints on the admissible set were removed, most of the reduced order trajectories required less than a minute of computation time with a crude initial guess. The program was capable of finding an optimal solution for a variety of initial and final conditions, performance index coefficients, initial guesses, and constraint formulations.

Of all the formulations tested, the formulation that gives the best results in terms of speed, reliability, and solution properties has hard constraints on $\theta\left(E_{f}\right)$, $\phi\left(E_{f}\right)$ and the two control rates. Due to the nonconvex shape of the admissible set, the constraints on the admissible set are best replaced with soft constraints. A hard constraint on the maximum drag boundary, is enforced as a redundant constraint to ensure that the trajectory does not violate this boundary of the admissible set. The soft constraint on the admissible set and soft constraints on the control rates smoothes the control trajectory such that rapid variations from one boundary of the admissible set to another do not occur. Having both hard and soft constraints on the control rate also reduces the number of bank reversals and eliminates the occurrence of short peaks in control rates.

The optimal reduced-order algorithm for 3D entry trajectory planning is applied to a model of a reusable second stage vehicle. The reusable second stage has a mass of $85000 \mathrm{lb}$, reference area of $1300 \mathrm{ft}^{2}$, maximum $C_{L}$ of 3.6 , and a maximum $L / D$ of 8.0 at an angle-of-attack of 10 degrees. For the numerical examples, baseline initial conditions of zero degrees longitude, latitude, and heading and a final destination of 12 degrees longitude and 6 degrees latitude are used. The coefficients given for the performance index, Eqs.(16) and (20), are $a_{1}=0, a_{2}=5.55 \times 10^{-4}$, $a_{3}=3.85 \times 10^{-4}, a_{4}=0.385, b_{1}=0.7, b_{2}=4$, and $b_{3}=0.7$. The initial/final altitudes and velocities are set to $r_{0}=2.7 \times 10^{+5} \mathrm{ft}, r_{f}=9.0 \times 10^{+4} \mathrm{ft}$, $V_{0}=17500 \mathrm{ft} / \mathrm{s}$, and $V_{f}=3000 \mathrm{ft} / \mathrm{s}$ and linearly interpolated to obtain the reference radius and energy. The constraints on normal acceleration, dynamic pressure, and maximum angle-of-attack are set to $q_{\max }=600$ $\mathrm{lb} / \mathrm{ft}^{2}, A_{\max }=128 \mathrm{ft} / \mathrm{s}^{2}, \alpha_{\max }=40$ degrees, and 
$\alpha_{\min }=\alpha$ for $(L / D)_{\max }$.

To illustrate the flexibility of the optimal reducedorder 3D trajectory planner, several trajectories are computed with different final heading angles. If the available energy is greater than the minimum required to achieve the specified longitude and altitude, a range of final heading angles can be reached, and it is possible to specify a final heading angle within this range, e.g., to align the vehicle with the runway. With the 2D Space Shuttle planner it is not possible to specify a desired heading angle. The optimal reduced-order $3 \mathrm{D}$ planning program allows the final heading to be specified. Figure 3 shows trajectories generated under the same conditions, except that various final heading angles are specified via a penalty term in the cost function. For this entry problem the feasible final heading angles range from -20 to 90 degrees. Trajectories with a shorter downrange requirement allow for a larger range of feasible heading angles. Short range trajectories have more energy available for maneuvering the vehicle to a wider range of final heading angles (Fig. 4).
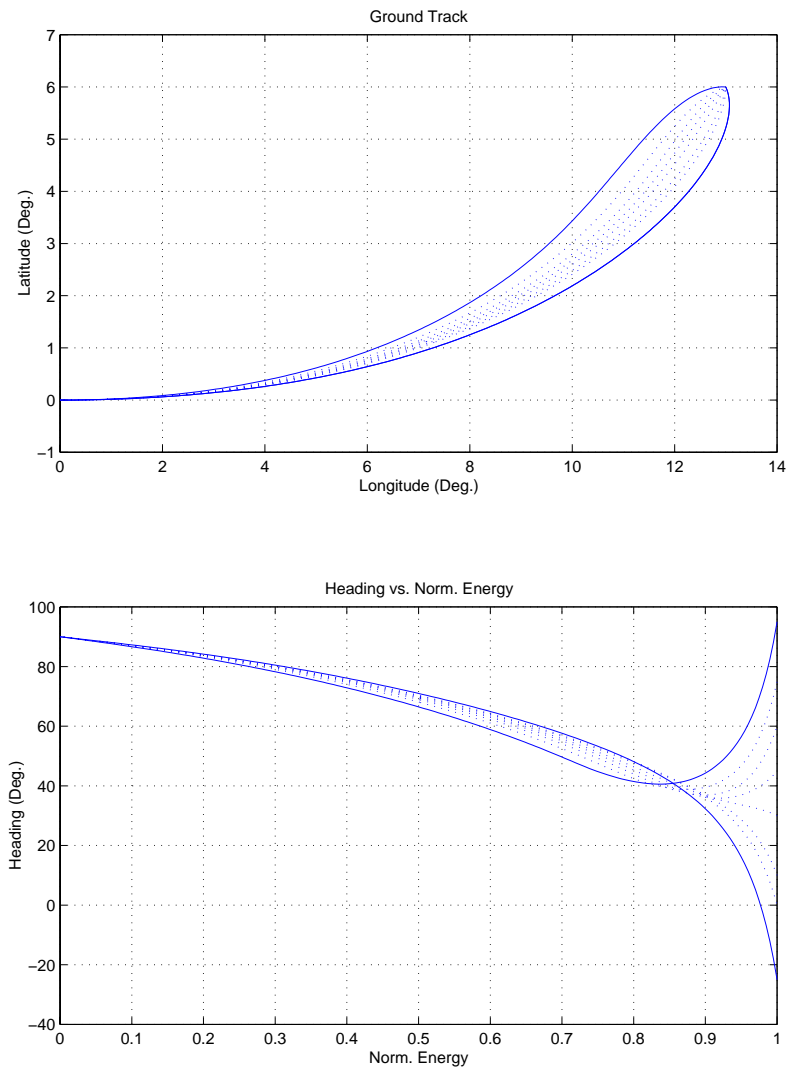

Fig. 3 Computed long-range trajectories for various final heading angles.

A similar analysis can be performed to determine the crossrange capability. Figure 5 shows various trajectories for a fixed downrange of 18 degrees. The crossrange varies from -7 to 7 degrees latitude.
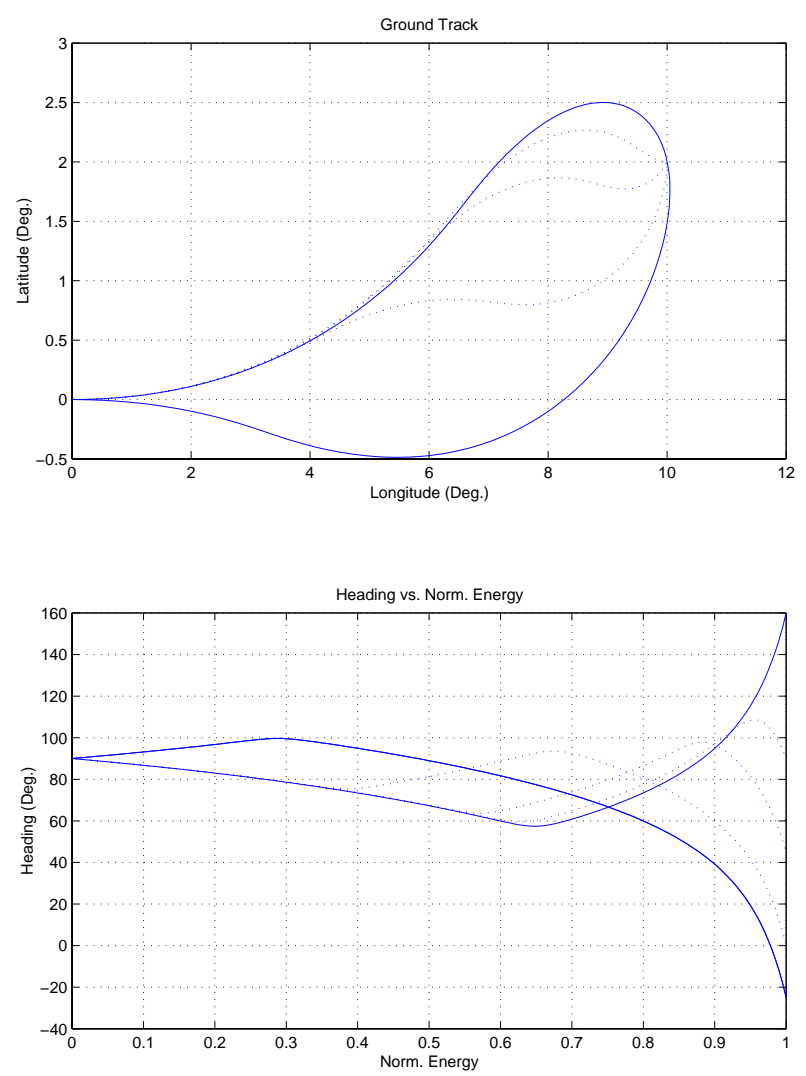

Fig. 4 Computed short-range trajectories for various final heading angles.
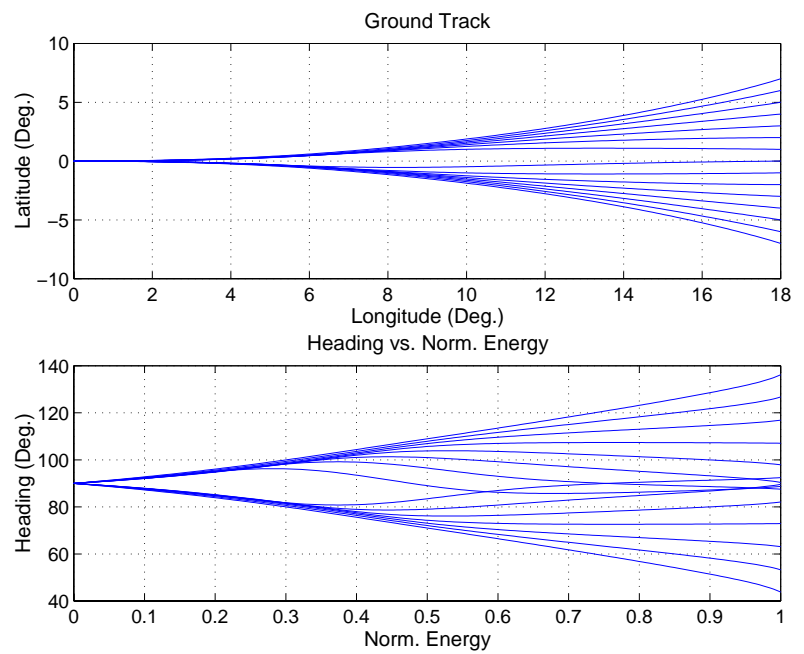

Fig. 5 Computed trajectories for various crossrange requirements.

\section{Comparison with OTIS Solution}

Figure 6 shows a comparison between entry trajectories computed by the optimal reduced-order planner and by OTIS. The vehicle and environmental models, boundary conditions, and dynamic pressure, acceleration, and heating rate constraints are the same. Both cost functions include heat load. The cost function 
for the reduced-order planner includes soft constraints to favor flight in the center of the entry corridor and to limit the rates of change of $D$ and $(L / D) \sin \sigma$. The cost function for OTIS has a soft constraint that limits the rate of change of angle-of-attack. These differences in the cost function cause differences in the corresponding solutions.

While the two trajectories reach the same target, the details of the trajectories and the control profiles used to obtain them have differences, especially in the latter part of the flight after the bank angle changes from negative to positive values. The results illustrate that, for a case like this when the available energy is greater than the minimum required to achieve the desired final state, there is more than one feasible trajectory. The trajectory generated by the optimal reduced-order planner has little phugoid-like oscillations, stays away from constraint boundaries, and does not require high control rates. These features are desirable for tracking.

The reduced-order planner only determines $\theta, \phi, \psi$, $D$, and $(L / D) \sin \sigma$. The other variables, $r, \gamma, \alpha$, and $\sigma$, have been extracted as described previously.

\section{Summary}

The Space Shuttle's 2D entry trajectory planning method has been extended to three dimensions. Both angle-of-attack and angle-of-bank variations are used to control the entry trajectory. The trajectory planning is performed with a third-order system of differential equations using the drag and lateral accelerations as intermediate controls. A feasible trajectory planning approach and an optimal trajectory planning approach have been discussed. Numerical results for the optimal reduced-order planning, including comparisons with optimal full-order solutions, illustrated the capabilities and attributes of the approach. 

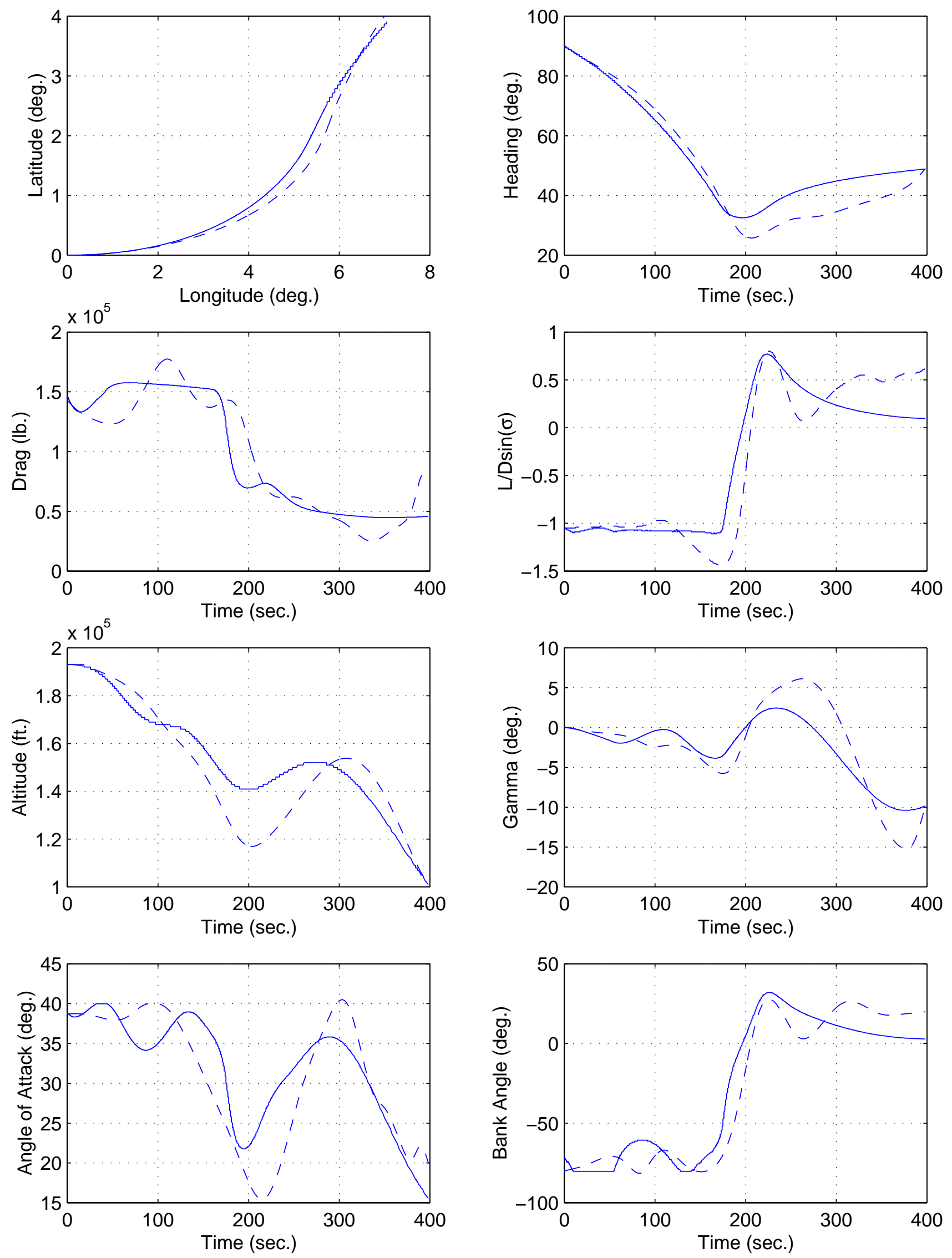

Fig. 6 Solutions from Reduced-Order Planning (solid line) and OTIS (dashed line) for Similar Problem 


\section{$\underline{\text { References }}$}

${ }^{1}$ Mease, K. D., Teufel, P., Schönenberger, H., Chen, D. T., Bharadwaj, S., "Re-Entry Trajectory Planning for a Reusable Launch Vehicle," Paper 99-4160, AIAA AFM-Conference, Portland, Aug. 1999.

${ }^{2}$ Harpold, J.D. and Graves, C.A., Jr., "Shuttle Entry Guidance," J. Astronautical Sciences, Vol. 27, No. 3, 1979, pp. 239-268.

${ }^{3}$ Mease, K. D. and Kremer, J.-P., "Shuttle Entry Guidance Revisited Using Nonlinear Geometric Methods," J. Guidance, Control and Dynamics, Nov.-Dec. 1994, pp. 1350-1356.

${ }^{4}$ Bharadwaj, S., Rao, A. V., and Mease, K. D., "Entry Trajectory Tracking Law via Feedback Linearization," J. Guidance, Control, and Dynamics, Sep.-Oct. 1998, pp. 726-732.

${ }^{5}$ Stengel, R. F., "Optimal Guidance for the Space Shuttle Transition," J. Spacecraft, Vol. 11, No. 3, 1974, pp. 173-179.

${ }^{6}$ Roenneke, A. J. and Markl, A., "Re-Entry Control to a Drag-vs-Energy Profile," J. Guidance, Control and Dynamics, Sep.-Oct. 1994, pp. 916-920.

${ }^{7}$ Vinh, N. X. Optimal Trajectories in Atmospheric Flight, Elsevier, New York, 1981.

${ }^{8} \mathrm{Lu}, \quad$ P., "Entry Guidance and Trajectory Control for Reusable Launch Vehicle," J. Guidance, Control, and Dynamics, Jan.-Feb. 1997, pp. 143-149.

${ }^{9}$ Martens, P. J., Paris, S. W., Hargraves, C. R., and Lajoie, R. M., "Optimal Trajectories by Implicit Simulation," WRDCTR-9-3056, Dec. 1990.

${ }^{10}$ Betts, J. T. and Huffman, W. P., Sparse Optimal Control Software, The Boeing Company, Seattle, 1997. 